QUARTERLY OF APPLIED MATHEMATICS

VOLUME LXVIII, NUMBER 3

SEPTEMBER 2010, PAGES 395-419

S 0033-569X(10)01199-9

Article electronically published on June 8, 2010

\title{
TWO STABLE-BY-HOMOGENIZATION MODELS IN SIMPLE SHEARING OF RATE-DEPENDENT NON-HOMOGENEOUS MATERIALS
}

\author{
BY \\ NICOLAS CHARALAMBAKIS (Department of Civil Engineering, Aristotle University, GR 54124 \\ Thessaloniki, Greece) \\ AND
}

FRANÇOIS MURAT (Laboratoire Jacques-Louis Lions, Université Pierre et Marie Curie, Boîte courrier 187, 75252 Paris Cedex 05, France)

\begin{abstract}
In this paper we study two models, the viscoplastic model and the thermoviscous model, of rate-dependent non-homogeneous materials with non-oscillating strainrate sensitivity submitted to simple quasistatic shearing. We prove that the two models are stable by homogenization, i.e. that the equations in both the heterogeneous problems and the homogenized one have the same form, and we give explicit formulas for the homogenized (effective) coefficients. These formulas depend on the initial conditions, but not on the boundary conditions. Our theoretical results are illustrated by a numerical example.
\end{abstract}

1. Introduction. Many methods have been proposed to provide "effective" equations of highly heterogeneous materials. Mathematical homogenization, which is adopted in the present paper, consists of setting the problem as a sequence of equations describing the heterogeneous material when the heterogeneities, whose typical size is characterized by a parameter $\varepsilon$, become smaller and smaller. Passing to the limit in the problem is a nonlinear process (even if the problem is linear) when both the solution and the coefficients are concerned. To quote only a very few works in this direction, we cite Tartar (1977), Murat (1977), Bensoussan et al. (1978), and Sanchez-Palencia (1978).

In terms used in mechanics, homogenization aims at replacing a highly heterogeneous material with an equivalent (effective) one. This method can of course be used to design new materials with enhanced properties. It has often been described in mechanics literature, and here again, giving a complete list is impossible; let us mention Suquet (1982), Francfort et al. (1983a), Francfort et al. (1983b), Hashin (1983), Suquet (1983),

Received November 21, 2007.

2000 Mathematics Subject Classification. Primary 74Q15, 74Q10, 35B27.

E-mail address: charalam@civil.auth.gr

E-mail address: murat@ann.jussieu.fr

(C) 2010 Brown University

Reverts to public domain 28 years from publication 
Maugin (1992), Aboudi et al. (1999), Ghosh et al. (2001), Bansal and Pindera (2003), Michel and Suquet (2004), Alshits and Maugin (2005), Bansal and Pindera (2005), Bardzokas and Zobnin (2005), Guinovart-Diaz et al. (2005), Suquet (2005), Batra and Love (2006a), Batra and Love (2006b), Idiart et al. (2006), Cavalcante et al. (2007), Cavalcante et al. (2008), as well as the excellent review paper by Pindera et al. (2009).

It would be interesting to look for the conditions under which a given model has the same form at the phases' and at the homogenized level. This allows one to look for effective parameters and simplifies the characterization of mechanical properties of heterogeneous materials. Here we define "stable-by-homogenization" ( $\mathrm{SbH}$ ) thermoviscoplastic models (i.e. models in which the equations in both the heterogeneous and the homogenized problem are of the same form). We show that the quasistatic shearing under dynamical conditions is $\mathrm{SbH}$ for two specific models of rate-dependent materials, the viscoplastic model and the thermoviscous model.

The simple shearing between the planes $x=a, x=b$ of a rigid rate-dependent material made of numerous layers of thickness of order $\varepsilon$ perpendicular to the $x$-direction, with different referential densities $\rho^{\varepsilon}$, specific heat coefficients $\eta^{\varepsilon}$, rates of plastic work converted into heat $\beta^{\varepsilon}$, strain-rate sensitivities $n^{\varepsilon}$, viscosity coefficients $\psi^{\varepsilon}$ and body force $f$, is described by the balance laws of the process and the compatibility equation, which relate the unknowns of the problem (namely the velocity $v^{\varepsilon}(t, x)$, the shear stress $\sigma^{\varepsilon}(t, x)$, the strain $\gamma^{\varepsilon}(t, x)$ and the temperature $\left.\theta^{\varepsilon}(t, x)\right)$, namely

$$
\begin{gathered}
\rho^{\varepsilon}(x) \frac{\partial v^{\varepsilon}}{\partial t}=\frac{\partial \sigma^{\varepsilon}}{\partial x}+f(t, x), \\
c^{\varepsilon}\left(x, \theta^{\varepsilon}\right) \frac{\partial \theta^{\varepsilon}}{\partial t}=\sigma^{\varepsilon} \frac{\partial v^{\varepsilon}}{\partial x}, \\
\frac{\partial \gamma^{\varepsilon}}{\partial t}=\frac{\partial v^{\varepsilon}}{\partial x},
\end{gathered}
$$

and by the constitutive law, which here will be assumed to be of the form

$$
\sigma^{\varepsilon}=\psi^{\varepsilon}\left(x, \gamma^{\varepsilon}, \theta^{\varepsilon}\right)\left|\frac{\partial v^{\varepsilon}}{\partial x}\right|^{n^{\varepsilon}(x)-1} \frac{\partial v^{\varepsilon}}{\partial x},
$$

while the heat coefficient $c^{\varepsilon}$ is defined by

$$
c^{\varepsilon}\left(x, \theta^{\varepsilon}\right)=\frac{\rho^{\varepsilon}(x) \eta^{\varepsilon}\left(x, \theta^{\varepsilon}\right)}{\beta^{\varepsilon}\left(x, \theta^{\varepsilon}\right)} .
$$

Here $t \in(0, T)$ and $x \in \Omega=(a, b)$.

The corresponding quasistatic problem is obtained by assuming that the inertial effects are negligible $\left(\frac{\partial v^{\varepsilon}}{\partial t} \sim 0\right)$. Then (1.1) reads as

$$
-\frac{\partial \sigma^{\varepsilon}}{\partial x}=f
$$

The above system (1.1) - 1.5. has, of course, to be complemented by suitable initial and boundary conditions. We note that all the (given) material functions $\rho^{\varepsilon}(x), c^{\varepsilon}(x, \theta)$, $n^{\varepsilon}(x)$ and $\psi^{\varepsilon}(x, \gamma, \theta)$ are only assumed to be bounded from above and from below by strictly positive constants but are allowed to exhibit discontinuities with respect to $x$, so 
that equations (1.1)-1.3) must be understood in the sense of distributions. Moreover, the heat coefficient $c^{\varepsilon}(x, \theta)$ and the viscosity coefficient $\psi^{\varepsilon}(x, \gamma, \theta)$ are supposed to be uniformly Lipschitz continuous with respect to $\gamma$ and $\theta$.

Homogenization consists of describing the overall behavior of the velocity $v^{\varepsilon}$, stress $\sigma^{\varepsilon}$, strain $\gamma^{\varepsilon}$ and temperature $\theta^{\varepsilon}$ for fixed external forces and initial and boundary conditions, which is expressed by the weak limits (in suitable spaces) $\left(v^{0}, \sigma^{0}, \gamma^{0}, \theta^{0}\right)$ of $\left(v^{\varepsilon}, \sigma^{\varepsilon}, \gamma^{\varepsilon}\right.$, $\left.\theta^{\varepsilon}\right)$ as $\varepsilon$ tends to zero.

When $n^{\varepsilon}(x)=1$ and $\psi^{\varepsilon}(x, \gamma, \theta)=\mu^{\varepsilon}\left(x, \theta^{\varepsilon}\right)$ in (1.4), and when $\frac{\partial \mu^{\varepsilon}}{\partial \theta} \leq 0$ holds, i.e. in the case of the special constitutive law

$$
\sigma^{\varepsilon}=\mu^{\varepsilon}\left(x, \theta^{\varepsilon}\right) \frac{\partial v^{\varepsilon}}{\partial x}
$$

with thermal softening, the dynamical problem (1.1)-(1.4) with Dirichlet or Neumann or mixed boundary conditions has been studied by the authors (Charalambakis and Murat (1989), Charalambakis and Murat (2006a)), and its homogenization has been presented in Charalambakis and Murat (2006b). In this setting, the dynamical problem (1.1)-(1.3), (1.7) is $\mathrm{SbH}$.

In the present paper, we concentrate on the quasistatic problem. We prove that the quasistatic problem is $\mathrm{SbH}$ if the constitutive law (1.4) (and, in some cases, the heat coefficient $c^{\varepsilon}$ ) takes special forms. More specifically, we prove that when $n^{\varepsilon}(x)=n^{\star}(x)$ and when either

(i) $\psi^{\varepsilon}(x, \gamma, \theta)=\nu^{\varepsilon}(x, \gamma)$ and $c^{\varepsilon}(x, \theta)=c^{\star}(x)$ (viscoplastic case), or when

(ii) $\psi^{\varepsilon}(x, \gamma, \theta)=\mu^{\varepsilon}(x, \theta)$ (thermoviscous case), the quasistatic problem (1.2)-(1.4), (1.6) is SbH. Recall that in Charalambakis and Murat (2009) we proved that the quasistatic problem (1.2)-(1.4), (1.6) is $\mathrm{SbH}$ when $\psi^{\varepsilon}(x, \gamma, \theta)$ is general but when $\sigma^{\varepsilon}(t, x)=\sigma^{\star \star}(x)$. Therefore the problem is $\mathrm{SbH}$ for (at least) the three following models: (i) the viscoplastic model, exhibiting strain-dependent viscosity $\nu^{\varepsilon}(x, \gamma)$, with non-oscillating strain-rate sensitivity $n^{\star}(x)$ and with temperatureindependent non-oscillating heat coefficient $c^{\star}(x)$; (ii) the thermoviscous model, exhibiting temperature-dependent viscosity $\mu^{\varepsilon}(x, \theta)$, with non-oscillating strain-rate sensitivity $n^{\star}(x)$ and with temperature-dependent heat coefficient $c^{\varepsilon}(x, \theta)$; (iii) the general thermoviscoplastic model $\psi^{\varepsilon}(x, \gamma, \theta)$, with non-oscillating strain-rate sensitivity $n^{\star}(x)$ and with temperature-dependent heat coefficient $c^{\varepsilon}(x, \theta)$, in the case where the material is sheared by steady boundary shearing and body force.

In the three models (i)-(iii), the homogenized coefficients depend on the initial values of the strain or of the temperature. This reflects the nonelastic character of the problem (1.2) -(1.4), (1.6). Moreover, in the case (iii), the homogenized viscosity $\psi^{0}$ and heat coefficient $c^{0}$ depend also on the boundary shearing and on the body force. In contrast, in the cases (i) and (ii), the constitutive law of the homogenized material does not depend on the boundary conditions. This fact provides an easy characterization of the mechanical behavior of the homogenized material and allows an easy exploitation of experimental data. Let us finally observe that the materials exhibiting oscillating strainrate sensitivities are not $\mathrm{SbH}$ in general (see Remark 3.7 below). 
Regarding the nature of the above three models, model (i) and model (iii) are used to describe steels under large strains, so it makes sense to investigate a given microstructure. It is true that model (ii) is essentially a fluid, in which case the microstructure is transported by the flow. However, in the special case of a solid in which the strain acts as a hidden variable in the viscosity function (for instance, when it can be expressed explicitly in terms of the temperature), the strain- and temperature-dependent viscosity function can be written as in the thermoviscous case (ii). This is, for instance, the case of a solid sheared by steady boundary stress with temperature-independent heat coefficient, as one may easily verify by integrating the energy equation (1.2) after using (1.3).

It is worth noticing that the above setting is very rigid, since the stability by homogenization depends heavily upon the fact that the stress and the strain rate sensitivity coefficient do not depend on $\varepsilon$, prohibiting any changes in the constitutive behavior (adding elasticity for example), or any change in the type of boundary conditions, and forcing the approximation of the rate dependence of constituents by a non-oscillating coefficient. Thus, the notion of stability by homogenization seems a bit ad hoc. However, our analysis allows for the explanation of some nonexpected effects arising from homogenization in thermoviscoplastic materials, such as the memory effects, like that of the effective viscosity coefficient exhibited in Remark 3.2, or like that of the time dependence of the loading conditions in model (iii). In this context, this one-dimensional setting could prove instructive as a case study and a guideline for future investigation.

The paper is organized as follows. In Section 2 we present an existence and uniqueness result for the general quasistatic problem which we proved in Charalambakis and Murat (2009), and we define the notion of stability by homogenization (SbH). In Sections 3 , 4 and 5 we prove that the system is $\mathrm{SbH}$ for two cases of materials: the viscoplastic case and the thermoviscous case. In Section 3 we also present a numerical example concerned with multiphase stratified materials with periodic structure when the phases are characterized by power laws; we observe that the homogenized material is no more characterized by a power law, and therefore that power laws are not $\mathrm{SbH}$.

2. Existence and uniqueness. In this section we consider the general quasistatic problem posed in $Q=(0, T) \times \Omega$, where $T>0$ and where $\Omega=(a, b)$ is one-dimensional, namely

$$
\begin{gathered}
-\frac{\partial \sigma^{\varepsilon}}{\partial x}=f \quad \text { in } Q, \\
\frac{\partial \gamma^{\varepsilon}}{\partial t}=\frac{\partial v^{\varepsilon}}{\partial x} \quad \text { in } Q, \\
c^{\varepsilon}\left(x, \theta^{\varepsilon}\right) \frac{\partial \theta^{\varepsilon}}{\partial t}=\sigma^{\varepsilon} \frac{\partial v^{\varepsilon}}{\partial x} \quad \text { in } Q, \\
\sigma^{\varepsilon}=\psi^{\varepsilon}\left(x, \gamma^{\varepsilon}, \theta^{\varepsilon}\right)\left|\frac{\partial v^{\varepsilon}}{\partial x}\right|^{n^{\varepsilon}(x)-1} \frac{\partial v^{\varepsilon}}{\partial x} \quad \text { in } Q,
\end{gathered}
$$


complemented by the initial conditions

$$
\begin{array}{ll}
\gamma^{\varepsilon}(0, x)=\gamma_{0}(x) & \text { in } \Omega, \\
\theta^{\varepsilon}(0, x)=\theta_{0}(x) & \text { in } \Omega,
\end{array}
$$

and by the boundary conditions

$$
\begin{aligned}
& \sigma^{\varepsilon}(t, a)=\sigma_{a}(t) \quad \text { in }(0, T), \\
& v^{\varepsilon}(t, b)=v_{b}(t) \quad \text { in }(0, T) .
\end{aligned}
$$

2.1. Existence and uniqueness. In this subsection, we recall the statement of the existence and uniqueness result which is proved in Charalambakis and Murat (2009). We make the following hypotheses on the data, where $0<M<+\infty, 0<\alpha \leq \beta<+\infty$ and $0<\bar{\alpha} \leq \bar{\beta}<+\infty$ are given:

$$
\begin{gathered}
f=-\frac{\partial g}{\partial x} \quad \text { in } Q, \text { where } g \in C^{0}(\bar{Q}), \\
x \rightarrow c^{\varepsilon}(x, \theta) \text { is measurable on } \Omega, \forall \theta \in \mathbf{R}, \\
\left|\frac{\partial c^{\varepsilon}}{\partial \theta}(x, \theta)\right| \leq M, \quad \text { a.e. } x \in \Omega, \forall \theta \in \mathbf{R}, \\
\alpha \leq c^{\varepsilon}(x, \theta) \leq \beta, \quad \text { a.e. } x \in \Omega, \forall \theta \in \mathbf{R}, \\
x \rightarrow \psi^{\varepsilon}(x, \gamma, \theta) \text { is measurable on } \Omega, \quad \forall \gamma \in \mathbf{R}, \quad \forall \theta \in \mathbf{R}, \\
\left|\frac{\partial \psi^{\varepsilon}}{\partial \gamma}(x, \theta, \gamma)\right| \leq M, \quad \text { a.e. } x \in \Omega, \forall \gamma \in \mathbf{R}, \forall \theta \in \mathbf{R}, \\
\left|\frac{\partial \psi^{\varepsilon}}{\partial \theta}(x, \theta, \gamma)\right| \leq M, \quad \text { a.e. } x \in \Omega, \forall \gamma \in \mathbf{R}, \forall \theta \in \mathbf{R}, \\
\alpha \leq \psi^{\varepsilon}(x, \gamma, \theta) \leq \beta, \quad \text { a.e. } x \in \Omega, \forall \gamma \in \mathbf{R}, \forall \theta \in \mathbf{R}, \\
n^{\varepsilon} \in L^{\infty}(\Omega), \\
\bar{\alpha} \leq n^{\varepsilon}(x) \leq \bar{\beta}, \quad \text { a.e. } x \in \Omega, \\
\gamma_{0} \in L^{\infty}(\Omega), \\
\theta_{0} \in L^{\infty}(\Omega), \\
\sigma_{a} \in C^{0}([0, T]), \\
v_{b} \in L^{\infty}(0, T) .
\end{gathered}
$$

When considering their regularity with respect to $x$, the only hypothesis made on the heat coefficient $c^{\varepsilon}$, on the viscosity coefficient $\psi^{\varepsilon}$ and on the strain-rate sensitivity $n^{\varepsilon}$ is that they are bounded from below and from above by strictly positive constants. More specifically, they are allowed to exhibit discontinuities with respect to the spatial variable $x$. The same is valid for the initial strain $\gamma_{0}$ and the initial temperature $\theta_{0}$. It is also worth noticing that we do not make any softening or hardening hypothesis on the material behavior, since the viscosity coefficient $\psi^{\varepsilon}$ may have a negative or positive or nonsigned derivative with respect to strain and/or temperature. 
Proposition 2.1 (Charalambakis and Murat (2009)). When hypotheses (2.9)-(2.22) hold true, there exists a unique solution $\left(\sigma^{\varepsilon}, v^{\varepsilon}, \gamma^{\varepsilon}, \theta^{\varepsilon}\right)$ of (2.1) -(2.8) which satisfies

$$
\begin{gathered}
\sigma^{\varepsilon}=\sigma^{\star} \quad \text { in } Q, \\
v^{\varepsilon} \in L^{\infty}(Q), \quad \frac{\partial v^{\varepsilon}}{\partial x} \in L^{\infty}(Q), \\
\gamma^{\varepsilon} \in L^{\infty}(Q), \quad \frac{\partial \gamma^{\varepsilon}}{\partial t} \in L^{\infty}(Q), \\
\theta^{\varepsilon} \in L^{\infty}(Q), \quad \frac{\partial \theta^{\varepsilon}}{\partial t} \in L^{\infty}(Q),
\end{gathered}
$$

where $\sigma^{\star} \in C^{0}(\bar{Q})$ is given by

$$
\sigma^{\star}(t, x)=\sigma_{a}(t)+g(t, x)-g(t, a) \quad \text { in } \bar{Q} .
$$

Moreover $\sigma^{\varepsilon}, v^{\varepsilon}, \frac{\partial v^{\varepsilon}}{\partial x}, \gamma^{\varepsilon}, \frac{\partial \gamma^{\varepsilon}}{\partial t}, \theta^{\varepsilon}$ and $\frac{\partial \theta^{\varepsilon}}{\partial t}$ are bounded in $L^{\infty}(Q)$ independently of $\varepsilon$.

The sketch of the proof is the following: We define a new temperature $\hat{\theta}=$ $\int_{\theta_{0}(x)}^{\theta} c^{\varepsilon}(x, s) d s$ and obtain from (2.3) that $\frac{\partial \hat{\theta}^{\varepsilon}}{\partial t}=\sigma^{*} \frac{\partial v^{\varepsilon}}{\partial x}$. Since $\psi^{\varepsilon}\left(x, \gamma^{\varepsilon}, \theta^{\varepsilon}\right)=$ $\hat{\psi^{\varepsilon}}\left(x, \gamma^{\varepsilon}, \hat{\theta^{\varepsilon}}\right)$, (2.4) gives $\frac{\partial v^{\varepsilon}}{\partial x}=\left[\frac{\sigma^{*}}{\hat{\psi}^{\varepsilon}\left(x, \gamma^{\varepsilon}, \hat{\theta}^{\varepsilon}\right)}\right]^{\frac{1}{n^{\varepsilon}}}$, then we replace in (2.2) and (2.3) and obtain a system of two ordinary differential equations of the form $\frac{\partial}{\partial t}\left(\gamma^{\varepsilon} \hat{\theta}^{\varepsilon}\right)^{T}=F^{\varepsilon}\left(x, \gamma^{\varepsilon}, \hat{\theta}^{\varepsilon}\right)$, for which existence and uniqueness are ensured.

2.2. Definition of the stability by homogenization $(S b H)$. Since we have $\sigma^{\varepsilon}=\sigma^{\star}$ for each $\varepsilon>0$ (see (2.23) $)$, we define $\sigma^{0}$ by

$$
\sigma^{0}=\sigma^{\star} \quad \text { in } Q,
$$

and we have for every $\varepsilon>0$

$$
\sigma^{\varepsilon}=\sigma^{0} \quad \text { in } Q
$$

On the other hand, in view of the a priori bounds on $v^{\varepsilon}, \frac{\partial v^{\varepsilon}}{\partial x}, \gamma^{\varepsilon}, \frac{\partial \gamma^{\varepsilon}}{\partial t}, \theta^{\varepsilon}$ and $\frac{\partial \theta^{\varepsilon}}{\partial t}$ obtained in Proposition 2.1 it is possible to extract a subsequence $\varepsilon^{\prime}$ such that, as $\varepsilon^{\prime}$ tends to zero,

$$
\begin{gathered}
v^{\varepsilon^{\prime}} \rightarrow v^{0} \quad \text { in } L^{\infty}(Q) \text { weak-star, } \\
\frac{\partial v^{\varepsilon^{\prime}}}{\partial x} \rightarrow \frac{\partial v^{0}}{\partial x} \text { in } L^{\infty}(Q) \text { weak-star, } \\
\gamma^{\varepsilon^{\prime}} \rightarrow \gamma^{0} \text { in } L^{\infty}(Q) \text { weak-star }, \\
\frac{\partial \gamma^{\varepsilon^{\prime}}}{\partial t} \rightarrow \frac{\partial \gamma^{0}}{\partial t} \text { in } L^{\infty}(Q) \text { weak-star, } \\
\theta^{\varepsilon^{\prime}} \rightarrow \theta^{0} \text { in } L^{\infty}(Q) \text { weak-star }, \\
\frac{\partial \theta^{\varepsilon^{\prime}}}{\partial t} \rightarrow \frac{\partial \theta^{0}}{\partial t} \quad \text { in } L^{\infty}(Q) \text { weak-star },
\end{gathered}
$$

for some $v^{0}, \gamma^{0}$ and $\theta^{0}$ which belong to $L^{\infty}(Q)$, such that $\frac{\partial v^{0}}{\partial x}, \frac{\partial \gamma^{0}}{\partial t}$ and $\frac{\partial \theta^{0}}{\partial t}$ belong to $L^{\infty}(Q)$. 
It is then easy to pass to the limit in the linear equations of problem (2.1)-(2.8), namely (2.1), (2.2) and (2.5)-(2.8), obtaining

$$
\begin{gathered}
-\frac{\partial \sigma^{0}}{\partial x}=f \quad \text { in } Q, \\
\frac{\partial \gamma^{0}}{\partial t}=\frac{\partial v^{0}}{\partial x} \quad \text { in } Q, \\
\gamma^{0}(0, x)=\gamma_{0}(x) \quad \text { in } \Omega, \\
\theta^{0}(0, x)=\theta_{0}(x) \quad \text { in } \Omega, \\
\sigma^{0}(t, a)=\sigma_{a}(t) \quad \text { in }(0, T), \\
v^{0}(t, b)=v_{b}(t) \quad \text { in }(0, T) .
\end{gathered}
$$

In contrast, it is absolutely not clear whether one can pass to the limit in the nonlinear equations (2.3) and (2.4), and obtain equations similar to (2.3) and (2.4), which would read as

$$
\begin{gathered}
c^{0}\left(x, \theta^{0}\right) \frac{\partial \theta^{0}}{\partial t}=\sigma^{0} \frac{\partial v^{0}}{\partial x} \quad \text { in } Q \\
\sigma^{0}=\psi^{0}\left(x, \gamma^{0}, \theta^{0}\right)\left|\frac{\partial v^{0}}{\partial x}\right|^{n^{0}(x)-1} \frac{\partial v^{0}}{\partial x} \quad \text { in } Q,
\end{gathered}
$$

for some homogenized coefficients $c^{0}(x, \theta)$ and $\psi^{0}(x, \gamma, \theta)$ and some strain-rate sensitivity $n^{0}(x)$.

This leads to the following definition (see Charalambakis and Murat (2009)).

Definition 2.2. Assume that hypotheses (2.9) $-(2.22)$ hold true and let $\left(\sigma^{\varepsilon}, v^{\varepsilon}, \gamma^{\varepsilon}, \theta^{\varepsilon}\right)$ be the unique solution of problem (2.1)-(2.8). The problem (2.1)-(2.8) is said to be Stable by Homogenization $(\mathrm{SbH})$ if there exists a subsequence $\varepsilon^{\prime}$, a heat coefficient $c^{0}(x, \theta)$, a viscosity coefficient $\psi^{0}(x, \gamma, \theta)$ and a strain-rate sensitivity $n^{0}(x)$ satisfying (2.10) $-(2.18)$ (with constants $M, \alpha, \beta, \bar{\alpha}$ and $\bar{\beta}$ possibly different) such that for this subsequence, convergences (2.30)-(2.35) hold true for the unique solution $\left(\sigma^{0}, v^{0}, \gamma^{0}, \theta^{0}\right)$ of problem (2.36)-(2.43) .

Then $c^{0}(x, \theta), \psi^{0}(x, \gamma, \theta)$ and $n^{0}(x)$ are called the homogenized heat coefficient, the homogenized viscosity coefficient and the homogenized strain-rate sensitivity of problem (2.1) (2.8), respectively.

Note that in Definition 2.2 the homogenized quantities $c^{0}(x, \theta), \psi^{0}(x, \gamma, \theta)$ and $n^{0}(x)$ can depend on the force $f$ and on the initial and boundary data $\gamma_{0}, \theta_{0}, \sigma_{a}$ and $v_{b}$. This is a consequence of the nonelastic character of problem (2.1)-(2.8).

In general, when only the general hypotheses (2.9)-(2.22) are made, problem (2.1)(2.8) is not $\mathrm{SbH}$ (see Remark 3.7 below). In contrast, we will prove in Sections 3 , 4 and 5 of the present paper that, for at least two special models, the system (2.1)-(2.8) is $\mathrm{SbH}$ (see Proposition 3.1 and Proposition 4.1). 
3. Problem (2.1) $-(2.8)$ is $\mathbf{S b H}$ when the viscosity coefficient $\psi^{\varepsilon}(x, \gamma, \theta)$ does not depend on the temperature $\theta$ (viscoplastic case).

Proposition 3.1. Assume that hypotheses (2.9) $-(2.22)$ hold true and, moreover, that

$$
\begin{gathered}
n^{\varepsilon}(x)=n^{\star}(x), \\
c^{\varepsilon}(x, \theta)=c^{\star}(x), \\
\psi^{\varepsilon}(x, \gamma, \theta)=\nu^{\varepsilon}(x, \gamma),
\end{gathered}
$$

for some given $n^{\star}(x), c^{\star}(x)$ and $\nu^{\varepsilon}(x, \gamma)$. Then problem (2.1)-(2.8) is $\mathrm{SbH}$ and one has

$$
\begin{gathered}
n^{0}(x)=n^{\star}(x), \\
c^{0}(x, \theta)=c^{\star}(x), \\
\psi^{0}(x, \gamma, \theta)=\nu^{0}(x, \gamma),
\end{gathered}
$$

where the homogenized viscosity coefficient $\nu^{0}(x, \gamma)$ depends only on the sequence $\nu^{\varepsilon}(x, \gamma)$, on the strain-rate sensitivity $n^{\star}(x)$ and on the initial strain $\gamma^{0}(x)$. The homogenized viscosity coefficient $\nu^{0}(x, \gamma)$ is described in Remark 3.2 below.

Proof of Proposition 3.1. Since here $c^{\varepsilon}(x, \theta)=c^{\star}(x)$ depends neither on $\varepsilon$ nor on $\theta$, it is straightforward to pass to the limit in equation (2.3), which in view of (3.2) and (2.23) reads as

obtaining

$$
c^{\star}(x) \frac{\partial \theta^{\varepsilon}}{\partial t}=\sigma^{\star}(t, x) \frac{\partial v^{\varepsilon}}{\partial x} \quad \text { in } Q
$$

$$
c^{\star}(x) \frac{\partial \theta^{0}}{\partial t}=\sigma^{\star}(t, x) \frac{\partial v^{0}}{\partial x} \quad \text { in } Q .
$$

The only problem is therefore to pass to the limit in the constitutive law (2.4), which thanks to (3.1), (3.3) and (2.23) reads here as

$$
\sigma^{\star}(t, x)=\nu^{\varepsilon}\left(x, \gamma^{\varepsilon}\right)\left|\frac{\partial v^{\varepsilon}}{\partial x}\right|^{n^{\star}(x)-1} \frac{\partial v^{\varepsilon}}{\partial x} \quad \text { in } Q,
$$

or, equivalently, as

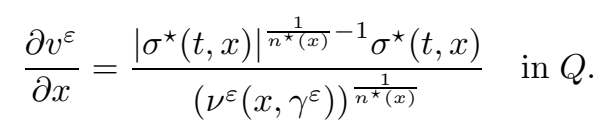

By (2.2), (2.5) and (3.7) we therefore have

$$
\begin{gathered}
\frac{\partial \gamma^{\varepsilon}}{\partial t}=\frac{\left|\sigma^{\star}(t, x)\right|^{\frac{1}{n^{\star}(x)}-1} \sigma^{\star}(t, x)}{\left(\nu^{\varepsilon}\left(x, \gamma^{\varepsilon}\right)\right)^{\frac{1}{n^{\star}(x)}}} \text { in } Q, \\
\gamma^{\varepsilon}(0, x)=\gamma_{0}(x) \quad \text { in } \Omega .
\end{gathered}
$$

For the rest of the proof, we refer the reader to Section 5, where the common proof of Propositions 3.1 and 4.1 is presented. In the next remarks of this section we give all useful results of the homogenization of the viscoplastic case.

REMARK 3.2. Definition of the homogenized viscosity coefficient $\nu^{0}(x, \gamma)$.

Let us summarize in this Remark the way in which $\nu^{0}(x, \gamma)$ is defined in the viscoplastic case considered in Proposition 3.1 . 
From the data $c^{\varepsilon}(x, s), \nu^{\varepsilon}(x, s), n^{\star}(x)$ and $\gamma_{0}(x)$, we define the function $\hat{Z}^{\varepsilon}(x, s)$ by

$$
\hat{Z}^{\varepsilon}(x, s)=\int_{\gamma_{0}(x)}^{s}\left(\nu^{\varepsilon}\left(x, s^{\prime}\right)\right)^{\frac{1}{n^{\star}(x)}} d s^{\prime} \quad \text { in } \Omega \times \mathbf{R}
$$

(see (5.6)). Then we extract a subsequence $\varepsilon^{\prime}$ such that the reciprocal functions $\left(\hat{Z}^{\varepsilon^{\prime}}\right)^{-1}(x, r)$ satisfy, for every $r \in \mathbf{R}$ fixed,

$$
\left(\hat{Z}^{\varepsilon^{\prime}}\right)^{-1}(x, r) \rightarrow\left(\hat{Z}^{0}\right)^{-1}(x, r) \text { in } L^{\infty}(\Omega) \text { weak-star, }
$$

for some function $\hat{Z}^{0}(x, s)$ (see (5.16) and (5.21)). From the same data, we also define (see (5.13), (5.17) and (5.23)) a function $\nu^{0}(x, s)$ such that (possibly for a further subsequence, still denoted by $\varepsilon^{\prime}$ ) one has, for every $r \in \mathbf{R}$ fixed,

$$
\frac{1}{\left(\nu^{\varepsilon^{\prime}}\left(x,\left(\hat{Z}^{\varepsilon^{\prime}}\right)^{-1}(x, r)\right)\right)^{\frac{1}{n^{\star}(x)}}}-\frac{1}{\left(\nu^{0}\left(x,\left(\hat{Z}^{0}\right)^{-1}(x, r)\right)\right)^{\frac{1}{n^{\star}(x)}}} \quad \text { in } L^{\infty}(\Omega) \text { weak-star. }
$$

This function $\nu^{0}(x, s)$ depends only (but does depend) on the sequence $\nu^{\varepsilon}(x, s)$, on the strain-rate sensitivity $n^{\star}(x)$ and on the initial condition $\gamma^{0}(x)$ (memory effect). It does not depend on the other data $\left(f, \theta_{0}, \sigma_{a}\right.$ and $\left.v_{b}\right)$ of the problem.

REMARK 3.3. The case of a multiphase viscoplastic heterogeneous material made of periodic homogeneous layers.

In this Remark we consider the special case of Proposition 3.1 where the heterogeneous viscoplastic material is made of periodic thin layers (of thickness of order $\varepsilon$ ) of homogeneous phases.

In other words, we consider here the case where

$$
\begin{gathered}
n^{\star}(x)=n^{\star \star}, \\
c^{\star}(x)=c^{\star \star}, \\
\nu^{\varepsilon}(x, \gamma)=\sum_{i} \chi_{i}\left(\frac{x}{\varepsilon}\right) \nu_{i}(\gamma),
\end{gathered}
$$

where $n^{\star \star}$ and $c^{\star \star}$ are given in $\mathbf{R}^{+}$, where the index $i$ runs between 1 and $I(I \geq 2$ denotes the number of phases), where $\nu_{i}: \mathbf{R} \rightarrow \mathbf{R}$ are viscosity coefficients which do not depend on $x$ (and therefore describe homogeneous phases) and which satisfy (2.14) and (2.16), where

$$
0=a_{0}<a_{1}<\ldots<a_{i-1}<a_{I}=1
$$

are given numbers and where $\chi_{i}$ is the characteristic function of the interval $\left(a_{i-1}, a_{i}\right)$ extended by periodicity to $\mathbf{R}$, i.e.

$$
\chi_{i}(x)= \begin{cases}1 & \text { if } k+a_{i-1}<x<k+a_{i} \text { for some } k \in \mathbf{Z}, \\ 0 & \text { otherwise. }\end{cases}
$$

We set

$$
p_{i}=a_{i}-a_{i-1} .
$$

The number $p_{i}$ describes the volume fraction of the phase $i$ in the material and satisfies

$$
p_{i}>0 \quad \forall i, \quad \sum_{i} p_{i}=1 .
$$


Observe that

$$
\chi_{i}\left(\frac{x}{\varepsilon}\right) \rightarrow p_{i} \quad \text { in } L^{\infty}(\Omega) \text { weak-star. }
$$

We finally assume that

$$
\gamma_{0}(x)=\gamma^{\star \star} \text { in } \Omega
$$

where $\gamma^{\star \star}$ is given in $\mathbf{R}$.

Hypotheses (2.9) $-(2.22)$ are then satisfied, and the present setting is a particular case of Proposition 3.1. In this setting, the function $\hat{Z}^{\varepsilon}$ defined by (5.6) is given by

$$
\hat{Z}^{\varepsilon}(x, s)=\sum_{i} \chi_{i}\left(\frac{x}{\varepsilon}\right) \int_{\gamma^{\star \star}}^{s}\left(\nu_{i}\left(s^{\prime}\right)\right)^{\frac{1}{n^{\star \star}}} d s^{\prime}=\sum_{i} \chi_{i}\left(\frac{x}{\varepsilon}\right) \hat{Z}_{i}(s),
$$

where $\hat{Z}_{i}: \mathbf{R} \rightarrow \mathbf{R}$ is the function defined by

$$
\hat{Z}_{i}(s)=\int_{\gamma^{\star \star}}^{s}\left(\nu_{i}\left(s^{\prime}\right)\right)^{\frac{1}{n^{\star \star}}} d s^{\prime} .
$$

Therefore the reciprocal function $\left(\hat{Z}^{\varepsilon}\right)^{-1}(x, r)$ is defined by

$$
\left(\hat{Z}^{\varepsilon}\right)^{-1}(x, r)=\sum_{i} \chi_{i}\left(\frac{x}{\varepsilon}\right)\left(\hat{Z}_{i}\right)^{-1}(r),
$$

where $\left(\hat{Z}_{i}\right)^{-1}: \mathbf{R} \rightarrow \mathbf{R}$ is the reciprocal function of the function $\hat{Z}_{i}$.

In view of (3.16) and (3.20), the function $\left(\hat{Z}^{0}\right)^{-1}(x, r)=Y^{0}(x, r)$, which is defined by (5.16) and (5.21), does not depend on $x$, and one has

$$
\left(\hat{Z}^{0}\right)^{-1}(r)=Y^{0}(r)=\sum_{i} p_{i}\left(\hat{Z}_{i}\right)^{-1}(r) .
$$

Similarly, the homogenized viscosity coefficient $\nu^{0}(x, s)$, which is defined by (5.13), (5.17) and (5.23), does not depend on $x$ : indeed, since the function $\pi^{\varepsilon}(x, r)$ defined by (5.13) is given here by

$$
\pi^{\varepsilon}(x, r)=\frac{1}{\left(\nu^{\varepsilon}\left(x,\left(\hat{Z}^{\varepsilon}\right)^{-1}(x, r)\right)\right)^{\frac{1}{n^{\star}(x)}}}=\sum_{i} \frac{\chi_{i}\left(\frac{x}{\varepsilon}\right)}{\left(\nu_{i}\left(\left(\hat{Z}_{i}\right)^{-1}(r)\right)\right)^{\frac{1}{n^{\star} \star}}},
$$

we deduce from (5.17), (5.21), (5.23) and (3.22) that

$$
\frac{1}{\left(\nu^{0}\left(\left(\hat{Z}^{0}\right)^{-1}(r)\right)\right)^{\frac{1}{n^{\star \star}}}}=\sum_{i} \frac{p_{i}}{\left(\nu_{i}\left(\left(\hat{Z}_{i}\right)^{-1}(r)\right)\right)^{\frac{1}{n^{\star \star}}}} .
$$

REMARK 3.4. The case of power laws for a multiphase viscoplastic heterogeneous material made of periodic layers.

Let us complete the previous Remark by an explicit example.

In the setting of Remark 3.3, consider the case where the heterogeneous viscoplastic material is made of periodic layers of $I$ phases with volume fractions $p_{i}$, which are characterized by viscosity coefficients $\nu_{i}(s)$ that satisfy hypotheses (2.14) and (2.16) and which are given by power laws in some interval $A \leq s \leq B$ of $\mathbf{R}^{+}$, i.e. which satisfy

$$
\nu_{i}(s)=G_{i} s^{m_{i}}, \quad \forall s \quad \text { with } \quad A \leq s \leq B,
$$


where $G_{i}$ are given in $\mathbf{R}^{+}$, where $m_{i}$ are given in $\mathbf{R}$ and where $A$ and $B$ are given with $0<A<B<+\infty$. Note that viscosity coefficients defined by power laws on the whole of $\mathbf{R}$ or even of $\mathbf{R}^{+}$would satisfy neither hypothesis (2.14) nor hypothesis (2.16); this is the reason why we assume that the viscosity coefficients $\nu_{i}(s)$ of the phases are given by power laws only in the interval $A \leq s \leq B$. On the other hand, since we can have $A=\delta$ and $B=1 / \delta$ with $\delta>0$ small, the viscosity coefficients $\nu_{i}(s)$ can be defined by power laws on a very large part of $\mathbf{R}^{+}$.

Note that in (3.24) the power laws could be replaced by exponential or logarithmic laws (see Lemaitre and Chaboche (2001), Wright (2002)). Let us also emphasize that the powers $m_{i}$ are not assumed to be positive or negative. This allows us to consider both softening and hardening processes, as well as problems with softening and hardening processes competing against each other.

We will finally assume that the powers $m_{i}$ and the initial strain $\gamma^{\star \star}$ satisfy

$$
\begin{gathered}
n^{\star \star}+m_{i} \neq 0 \quad \forall i, \\
\gamma^{\star \star} \leq A .
\end{gathered}
$$

These two hypotheses are not essential.

In this example the function $\hat{Z}_{i}(s)$ defined by (3.19) is given by

$$
\hat{Z}_{i}(s)=g_{i} s^{\frac{n^{\star \star}+m_{i}}{n^{\star \star}}}-\kappa_{i}, \quad \forall s \quad \text { with } \quad A \leq s \leq B
$$

(in the case where hypothesis (3.25) does not hold true, the power has to be replaced by a logarithm), where the constants $g_{i}$ and $\kappa_{i}$ are given by

$$
\begin{gathered}
g_{i}=\frac{n^{\star \star}}{n^{\star \star}+m_{i}} G_{i}^{\frac{1}{n^{\star \star}}}, \\
\kappa_{i}=g_{i} A^{\frac{n^{\star \star}+m_{i}}{n^{\star \star}}}-\int_{\gamma^{\star \star}}^{A}\left(\nu_{i}\left(s^{\prime}\right)\right)^{\frac{1}{n^{\star \star}}} d s^{\prime} .
\end{gathered}
$$

Note that

$$
\kappa_{i}=g_{i} A^{\frac{n^{\star \star}+m_{i}}{n^{\star \star}}} \text { when } A=\gamma^{\star \star} .
$$

The reciprocal function $\left(\hat{Z}_{i}\right)^{-1}(r)$ therefore satisfies

$$
\left(\hat{Z}_{i}\right)^{-1}(r)=\left(\frac{1}{g_{i}}\left(r+\kappa_{i}\right)\right)^{\frac{n^{\star \star}}{n^{\star}+m_{i}}}, \quad \forall r \quad \text { with } \quad \hat{Z}_{i}(A) \leq r \leq \hat{Z}_{i}(B) .
$$

Since the function $\hat{Z}_{i}(s)$ is strictly increasing and since $\hat{Z}_{i}\left(\gamma^{\star \star}\right)=0$, hypothesis (3.26) implies that $0 \leq \hat{Z}_{i}(A)<\hat{Z}_{i}(B)<+\infty$. We will assume, moreover, that there exist $C$ and $D$ such that

$$
\max _{i} \hat{Z}_{i}(A) \leq C<D \leq \min _{i} \hat{Z}_{i}(B) .
$$

Then on the interval $C \leq r \leq D$, the function $\left(\hat{Z}^{0}\right)^{-1}(r)=Y^{0}(r)$ defined by (3.21) is given by

$$
\left(\hat{Z}^{0}\right)^{-1}(r)=Y^{0}(r)=\sum_{i} p_{i}\left(\frac{1}{g_{i}}\left(r+\kappa_{i}\right)\right)^{\frac{n^{\star \star}}{n^{\star \star}+m_{i}}}, \quad \forall r \quad \text { with } \quad C \leq r \leq D,
$$


and formula (3.23) which defines the homogenized viscosity coefficient $\nu^{0}$ reads as

$$
\frac{1}{\left(\nu^{0}\left(Y^{0}(r)\right)\right)^{\frac{1}{n^{\star \star}}}}=\sum_{i} \frac{p_{i}}{G_{i}^{\frac{1}{n^{\star \star}}}\left(\frac{1}{g_{i}}\left(r+\kappa_{i}\right)\right)^{\frac{m_{i}}{n^{\star \star}+m_{i}}}}, \quad \forall r \quad \text { with } \quad C \leq r \leq D .
$$

Observe that power laws are not $\mathrm{SbH}$.

REMARK 3.5. A numerical example.

We now present, in the context of Remark 3.4 some numerical results concerning the homogenized viscosity coefficient of a bimetallic material (so $I=2$ here) exhibiting strain hardening (so $m_{i}>0$ here) and strain-rate sensitivity $n^{\star \star}>0$. In this case the constitutive law (2.4) reads as

$$
\sigma=\sum_{i=1}^{2} \chi_{i}\left(\frac{x}{\varepsilon}\right) G_{i} \gamma^{m_{i}}\left|\frac{\partial v}{\partial x}\right|^{n^{\star \star}-1} \frac{\partial v}{\partial x}, \quad \forall \gamma \quad \text { with } \quad A \leq \gamma \leq B
$$

for some $A$ and $B$ given with $0<A<B<+\infty$ (see (3.24)). We moreover assume that the initial strain satisfies

$$
\gamma^{\star \star}=A .
$$

Formulas (3.34) and (3.33) which define the homogenized viscosity coefficient $\nu^{0}$, together with (3.28) and (3.30), give

$$
\begin{aligned}
& \frac{1}{\left(\nu^{0}\left(Y^{0}(r)\right)\right)^{\frac{1}{n^{\star \star}}}}=\sum_{i=1}^{2} \frac{p_{i}}{G_{i}^{\frac{1}{n^{\star \star}+m_{i}}}\left(\frac{n^{\star \star}+m_{i}}{n^{\star \star}} r+G_{i}^{\frac{1}{n^{\star \star}}}\left(\gamma^{\star \star}\right)^{\frac{n^{\star \star}+m_{i}}{n^{\star \star}}}\right)^{\frac{m_{i}}{n^{\star \star}+m_{i}}}}, \\
& Y^{0}(r)=\sum_{i=1}^{2} \frac{p_{i}}{G_{i}^{\frac{1}{n^{\star \star}+m_{i}}}}\left(\frac{n^{\star \star}+m_{i}}{n^{\star \star}} r+G_{i}^{\frac{1}{n^{\star \star}}}\left(\gamma^{\star \star}\right)^{\frac{n^{\star \star}+m_{i}}{n^{\star \star}}}\right)^{\frac{n^{\star \star}}{n^{\star \star}+m_{i}}}, \\
& \forall r \quad \text { with } C \leq r \leq D .
\end{aligned}
$$

We consider a layered material made of two equally distributed phases (so that $p_{1}=$ $\left.p_{2}=\frac{1}{2}\right)$ which are different steels, with (Lemaitre and Chaboche (2001)

$$
\begin{array}{llll}
G_{1}=762 & \mathrm{MPa}, & m_{1}=0.167, & n_{1}=0.07 \\
G_{2}=962 & \mathrm{MPa}, & m_{2}=0.187, & n_{2}=0.07
\end{array}
$$

for different given values of the initial strain $\gamma^{\star \star}$.

Figures 1 and 2 present numerical results due to George Chatzigeorgiou, whose collaboration is gratefully acknowledged. Both figures present the values of the viscosity coefficients $\nu_{1}(r)$ and $\nu_{2}(r)$ for four values of the initial strain $\gamma^{\star \star}$ and the values of the homogenized viscosity coefficient $\nu^{0}(r)$ for the same values of $\gamma^{\star \star}$. The two figures only differ by the range considered for $r$. Contrarily to Figure 2 (onset of deformation), Figure 1 shows that, for large values of $r$, the homogenized viscosity coefficient does not depend in practice on the initial value of strain. Finally, it is worth noticing that the homogenized viscosity coefficient is more "attracted" by the hardening of the weaker material than by the hardening of the stronger one, in a ratio $2 / 1$. Indeed, due to 


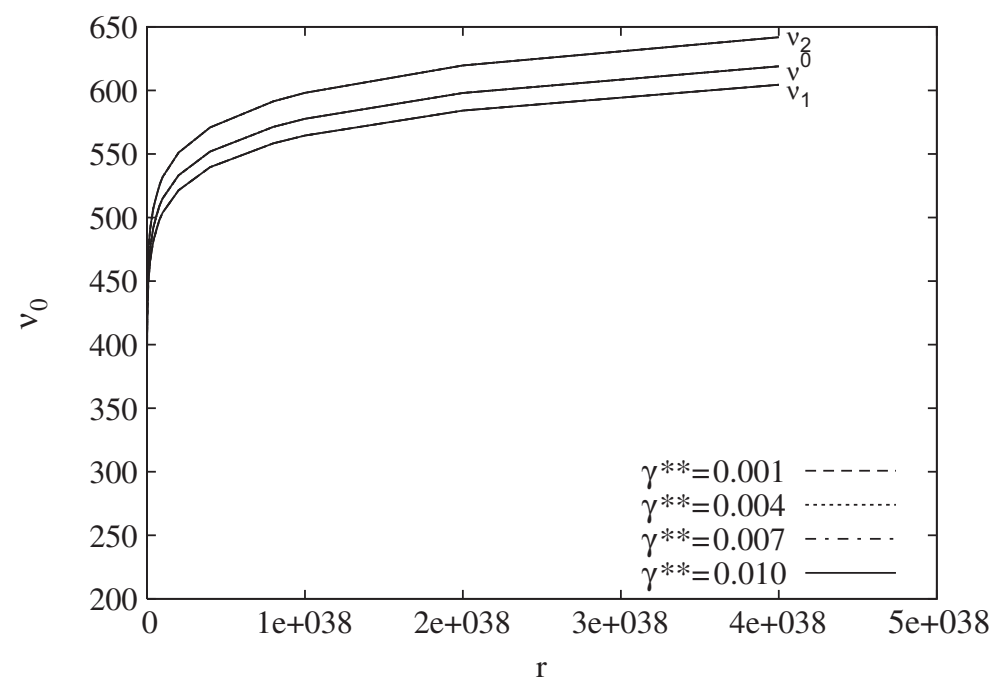

FIG. 1. The hardenings $\nu_{1}$ and $\nu_{2}$ of the phases and the homogenized hardening $\nu^{0}$ for different values of the initial strain $\gamma^{\star \star}$ (the curves seem to coincide for the four values of $\gamma^{\star \star}$ )

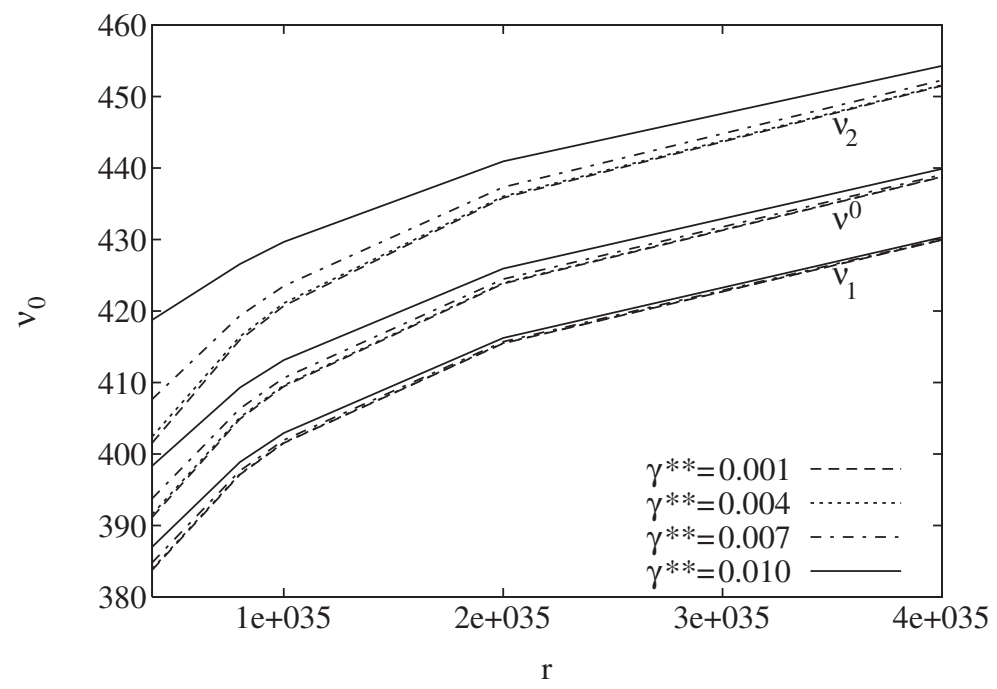

FIG. 2. The hardenings $\nu_{1}$ and $\nu_{2}$ of the phases and the homogenized hardening $\nu^{0}$ for different values of the initial strain $\gamma^{\star \star}$ at the onset of the deformation (the curves differ for the four values of $\gamma^{\star \star}$ )

the one-dimensional setting, the contribution of the strongest material is less important, because of the fact that one cannot "surround" the weakest material by the strongest one, in contrast with what can be done in a two- or three-dimensional setting (see also Charalambakis and Murat (2009)).

REMARK 3.6. A comment on the hypothesis $c^{\varepsilon}(x, \theta)=c^{\star}(x)$. 
In Proposition 3.1, the heat coefficient $c^{\varepsilon}(x, \theta)$ is assumed to be independent of $\varepsilon$ and of $x$ (see hypothesis (3.2)). One can wonder whether this hypothesis is necessary, i.e. whether one could pass to the limit in equation (2.3) when the heat coefficient is of the general form $c^{\varepsilon}(x, \theta)$.

Thanks to (2.23), equation (2.3) reads as

$$
c^{\varepsilon}\left(x, \theta^{\varepsilon}\right) \frac{\partial \theta^{\varepsilon}}{\partial t}=\sigma^{\star} \frac{\partial v^{\varepsilon}}{\partial x} \quad \text { in } Q .
$$

Defining the function $C^{\varepsilon}: \Omega \times \mathbf{R} \rightarrow \mathbf{R}$ by

$$
C^{\varepsilon}(x, s)=\int_{\theta_{0}(x)}^{s} c^{\varepsilon}\left(x, s^{\prime}\right) d s^{\prime} \quad \text { in } \Omega \times \mathbf{R},
$$

and defining the transformed temperature $\tau^{\varepsilon}=\tau^{\varepsilon}(t, x)$ by

$$
\tau^{\varepsilon}(t, x)=C^{\varepsilon}\left(x, \theta^{\varepsilon}(t, x)\right) \text { in } Q,
$$

equation (3.40) reads as

$$
\frac{\partial \tau^{\varepsilon}}{\partial t}=\sigma^{\star} \frac{\partial v^{\varepsilon}}{\partial x} \quad \text { in } Q
$$

while the initial condition (2.6) on $\theta^{\varepsilon}$ yields

$$
\tau^{\varepsilon}(0, x)=0 \quad \text { in } \Omega .
$$

Combining (3.42) and (3.43) with the convergence (2.31) implies that one can pass to the limit in (3.42) and (3.43) in $L^{\infty}(Q)$ weak-star, which defines in a unique way the limit $\tau^{0}$ of the transformed temperature $\tau^{\varepsilon^{\prime}}$. But since $\tau^{\varepsilon^{\prime}}$ and $\theta^{\varepsilon^{\prime}}$ converge only weakly, one cannot pass to the limit in the relation (3.41) and deduce a relation between $\theta^{0}$ defined by (2.34) and $\tau^{0}$. This is impossible even in the case where $c^{\varepsilon}(x, \theta)=c^{\varepsilon}(x)$ does not depend on $\theta$. This is the reason which forces us to assume hypothesis (3.2), namely that $c^{\varepsilon}(x, \theta)$ is independent of $\theta$ and of $\varepsilon$.

REMARK 3.7. Materials with oscillating strain-rate sensitivities are not SbH in general.

In this Remark, we prove by means of a counterexample that materials exhibiting oscillating strain-rate sensitivities are not $\mathrm{SbH}$ in general.

To build this counterexample, we consider the case where hypotheses (2.9)- (2.22) hold true and where

$$
\begin{gathered}
c^{\varepsilon}(x, \theta)=c^{\star}(x), \\
\psi^{\varepsilon}(x, \gamma, \theta)=\psi^{\star}(x), \\
\sigma^{\star}(t, x)=\sigma^{\star \star}(x),
\end{gathered}
$$

for some given $c^{\star}(x), \psi^{\star}(x)$ and $\sigma^{\star \star}(x)$. We moreover assume, as in Remark 3.3, that the material is made of periodic thin layers of homogeneous phases, namely that

$$
n^{\varepsilon}(x)=\sum_{i} \chi_{i}\left(\frac{x}{\varepsilon}\right) n_{i},
$$

for some given $n_{i}$, where the functions $\chi_{i}$ are defined by (3.14). Then equation (2.4) is equivalent to

$$
\frac{\partial v^{\varepsilon}}{\partial x}=\sum_{i} \chi_{i}\left(\frac{x}{\varepsilon}\right) \frac{\left|\sigma^{\star \star}(x)\right|^{\frac{1}{n_{i}-1}} \sigma^{\star \star}(x)}{\left(\psi^{\star}(x)\right)^{\frac{1}{n_{i}}}} \quad \text { in } Q
$$


and it is easy to pass to the limit in (2.2), (2.3), (2.5), (2.6) and (3.48), obtaining

$$
\begin{gathered}
\frac{\partial \gamma^{0}}{\partial t}=\frac{\partial v^{0}}{\partial x} \quad \text { in } Q, \\
c^{\star}(x) \frac{\partial \theta^{0}}{\partial t}=\sigma^{\star \star}(x) \frac{\partial v^{0}}{\partial x} \quad \text { in } Q, \\
\gamma^{0}(0, x)=\gamma_{0}(x) \quad \text { in } \Omega, \\
\theta^{0}(0, x)=\theta_{0}(x) \quad \text { in } \Omega, \\
\frac{\partial v^{0}}{\partial x}=\sum_{i} p_{i} \frac{\left|\sigma^{\star \star}(x)\right|^{\frac{1}{n_{i}-1}} \sigma^{\star \star}(x)}{\left(\psi^{\star}(x)\right)^{\frac{1}{n_{i}}}} \text { in } Q .
\end{gathered}
$$

Since $\sigma^{\star \star}$ does not depend on $t$, such is also the case of $\frac{\partial v^{0}}{\partial x}$ in view of (3.53), and (3.49) $-(3.52)$ imply that

$$
\begin{aligned}
\gamma^{0}(t, x) & =t \frac{\partial v^{0}}{\partial x}(x)+\gamma_{0}(x) \quad \text { in } Q, \\
c^{\star}(x) \theta^{0}(t, x) & =t \sigma^{\star \star}(x) \frac{\partial v^{0}}{\partial x}(x)+\theta_{0}(x) \quad \text { in } Q
\end{aligned}
$$

(this is in order to obtain these explicit formulas on $\gamma^{0}(t, x)$ and $\theta^{0}(t, x)$ that we made on $\sigma^{\star}$ for hypothesis (3.46) $)$.

We now claim that there does not exist any viscosity coefficient $\psi^{0}(x, \gamma, \theta)$ or strainrate sensitivity $n^{0}(x)$ such that

$$
\sigma^{0}=\psi^{0}\left(x, \gamma^{0}, \theta^{0}\right)\left|\frac{\partial v^{0}}{\partial x}\right|^{n^{0}(x)-1} \frac{\partial v^{0}}{\partial x} \text { in } Q .
$$

Indeed since $\sigma^{0}=\sigma^{\star \star}$ and $\frac{\partial v^{0}}{\partial x}$ do not depend on $t$, while $\gamma^{0}(t, x)$ and $\theta^{0}(t, x)$ are affine in $t$ by (3.54) and (3.55), formula (3.56) would imply that $\psi^{0}(x, \gamma, \theta)=\hat{\psi}(x)$ for some function $\hat{\psi}(x)$, and (3.56) would be equivalent to

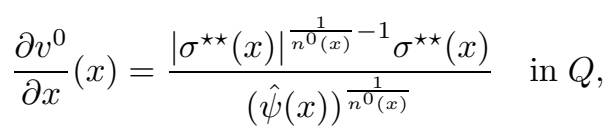

a contradiction with (3.53) since $\sigma^{\star \star}$ is arbitrary.

This counterexample proves that materials exhibiting oscillating strain-rate sensitivities are not $\mathrm{SbH}$ in general.

4. Problem (2.1) $-(2.8)$ is $\mathbf{S b H}$ when the viscosity coefficient $\psi^{\varepsilon}(x, \gamma, \theta)$ does not depend on the strain $\gamma$ (thermoviscous case).

Proposition 4.1. Assume that hypotheses (2.9)-(2.22) hold true and, moreover, that

$$
\begin{gathered}
n^{\varepsilon}(x)=n^{\star}(x), \\
\psi^{\varepsilon}(x, \gamma, \theta)=\mu^{\varepsilon}(x, \theta),
\end{gathered}
$$


for some given $n^{\star}(x)$ and $\mu^{\varepsilon}(x, \theta)$. Then problem (2.1)-(2.8) is $\mathrm{SbH}$ and one has

$$
\begin{gathered}
n^{0}(x)=n^{\star}(x), \\
\psi^{0}(x, \gamma, \theta)=\mu^{0}(x, \theta),
\end{gathered}
$$

where the homogenized heat coefficient $c^{0}(x, \theta)$ and the homogenized viscosity coefficient $\mu^{0}(x, \theta)$ depend only on the sequences $c^{\varepsilon}(x, \theta)$ and $\mu^{\varepsilon}(x, \theta)$, on the strain-rate sensitivity $n^{\star}(x)$ and on the initial temperature $\theta^{0}(x)$. The homogenized coefficients $c^{0}(x, \theta)$ and $\mu^{0}(x, \theta)$ are described in Remark 4.2 below.

Proof of Proposition 4.1. Here the problem is to pass to the limit in (2.3) and (2.4). In view of (2.23), equation (2.4) is here equivalent to

$$
\frac{\partial v^{\varepsilon}}{\partial x}=\frac{\left|\sigma^{\star}(t, x)\right|^{\frac{1}{n^{\star}(x)}-1} \sigma^{\star}(t, x)}{\left(\mu^{\varepsilon}\left(x, \theta^{\varepsilon}\right)\right)^{\frac{1}{n^{\star}(x)}}} \quad \text { in } Q,
$$

and therefore (2.3) reads as

$$
c^{\varepsilon}\left(x, \theta^{\varepsilon}\right) \frac{\partial \theta^{\varepsilon}}{\partial t}=\frac{\left|\sigma^{\star}(t, x)\right|^{\frac{1}{n^{\star}(x)}+1}}{\left(\mu^{\varepsilon}\left(x, \theta^{\varepsilon}\right)\right)^{\frac{1}{n^{\star}(x)}}} \quad \text { in } Q .
$$

For the rest of the proof, we refer the reader to Section 5, where the common proof of Propositions 3.1 and 4.1 is presented. In the following remarks we present useful results from the homogenization of thermoviscous materials.

Remark 4.2. Definition of the homogenized heat and viscosity coefficients $c^{0}(x, \theta)$ and $\mu^{0}(x, \theta)$.

Let us summarize in this Remark the way in which the homogenized coefficients $c^{0}(x, \theta)$ and $\mu^{0}(x, \theta)$ are defined in the thermoviscous case considered in Proposition 4.1

From the data $c^{\varepsilon}(x, s), \mu^{\varepsilon}(x, s), n^{\star}(x)$ and $\theta_{0}(x)$, we define the function $\hat{Z}^{\varepsilon}(x, s)$ by

$$
\hat{Z}^{\varepsilon}(x, s)=\int_{\theta_{0}(x)}^{s} c^{\varepsilon}\left(x, s^{\prime}\right)\left(\mu^{\varepsilon}\left(x, s^{\prime}\right)\right)^{\frac{1}{n^{\star}(x)}} d s^{\prime} \quad \text { in } \Omega \times \mathbf{R}
$$

(see (5.6) $)$. Then we extract a subsequence $\varepsilon^{\prime}$ such that the reciprocal functions $\left(\hat{Z}^{\varepsilon^{\prime}}\right)^{-1}(x, r)$ satisfy for every $r \in \mathbf{R}$ fixed

$$
\left(\hat{Z}^{\varepsilon^{\prime}}\right)^{-1}(x, r) \rightarrow\left(\hat{Z}^{0}\right)^{-1}(x, r) \text { in } L^{\infty}(\Omega) \text { weak-star, }
$$

for some function $\hat{Z}^{0}(x, s)$ (see (5.16) and (5.21)). From the same data, we also define (see (5.13), (5.17) and (5.23)) a function $\mu^{0}(x, s)$ such that (possibly for a further subsequence, still denoted by $\varepsilon^{\prime}$ ) one has for every $r \in \mathbf{R}$ fixed

$$
\frac{1}{\left(\mu^{\varepsilon^{\prime}}\left(x,\left(\hat{Z}^{\varepsilon^{\prime}}\right)^{-1}(x, r)\right)\right)^{\frac{1}{n^{\star}(x)}}} \rightarrow \frac{1}{\left(\mu^{0}\left(x,\left(\hat{Z}^{0}\right)^{-1}(x, r)\right)\right)^{\frac{1}{n^{\star}(x)}}} \quad \text { in } L^{\infty}(\Omega) \text { weak-star. }
$$

Finally from the functions $\hat{Z}^{0}(x, s)$ and $\mu^{0}(x, s)$, we define a function $c^{0}(x, s)$ by (5.28), i.e. by

$$
c^{0}(x, s)=\frac{\frac{\partial \hat{Z}^{0}}{\partial s}(x, s)}{\left(\mu^{0}(x, s)\right)^{\frac{1}{n^{\star}(x)}}} \quad \text { in } \Omega \times \mathbf{R}
$$


(an equivalent formula for $c^{0}(x, s)$ is (5.31)). The functions $\mu^{0}(x, s)$ and $c^{0}(x, s)$ depend only (but do depend) on the sequences $\mu^{\varepsilon}(x, s)$ and $c^{\varepsilon}(x, s)$, on the strain-rate sensitivity $n^{\star}(x)$ and on the initial condition $\theta_{0}(x)$. They do not depend on the other data $\left(f, \gamma_{0}, \sigma_{a}\right.$ and $v_{b}$ ) of the problem.

REMARK 4.3. The case of a multiphase thermoviscous heterogeneous material made of periodic homogeneous layers.

We consider here the case where (part of the notation in this Remark is the same as the notation in Remark 3.3 above)

$$
\begin{gathered}
n^{\star}(x)=n^{\star \star}, \\
c^{\varepsilon}(x, \theta)=\sum_{i} \chi_{i}\left(\frac{x}{\varepsilon}\right) c_{i}(\theta), \\
\mu^{\varepsilon}(x, \theta)=\sum_{i} \chi_{i}\left(\frac{x}{\varepsilon}\right) \mu_{i}(\theta),
\end{gathered}
$$

where $n^{\star \star}$ is given in $\mathbf{R}^{+}$, where the index $i$ runs between 1 and $I$ ( $I \geq 2$ denotes the number of phases), where $c_{i}: \mathbf{R} \rightarrow \mathbf{R}$ and $\mu_{i}: \mathbf{R} \rightarrow \mathbf{R}$ are heat and viscosity coefficients that do not depend on $x$ (and therefore describe homogeneous phases) and which satisfy respectively (2.11), (2.12) and (2.15), (2.16), where $\chi_{i}$ is the characteristic function defined in Remark 3.3. We assume that

$$
\theta_{0}(x)=\theta^{\star \star} \text { in } \Omega
$$

where $\theta^{\star \star}$ is given in $\mathbf{R}$.

Following parallel lines with Remark 3.3 we find

$$
\begin{gathered}
\hat{Z}_{i}(s)=\int_{\theta^{\star \star}}^{s} c_{i}\left(s^{\prime}\right)\left(\mu_{i}\left(s^{\prime}\right)\right)^{\frac{1}{n^{\star \star}}} d s^{\prime}, \\
\left(\hat{Z}^{\varepsilon}\right)^{-1}(x, r)=\sum_{i} \chi_{i}\left(\frac{x}{\varepsilon}\right)\left(\hat{Z}_{i}\right)^{-1}(r), \\
\frac{1}{\left(\mu^{0}\left(\hat{Y}^{0}(r)\right)\right)^{\frac{1}{n^{\star \star}}}}=\sum_{i} \frac{p_{i}}{\left(\mu_{i}\left(\left(\hat{Z}_{i}\right)^{-1}(r)\right)\right)^{\frac{1}{n^{\star \star}}}}
\end{gathered}
$$

and

$$
c^{0}\left(\hat{Y}^{0}(r)\right)=\frac{\sum_{i} \frac{p_{i}}{\left(\mu_{i}\left(\left(\hat{Z}_{i}\right)^{-1}(r)\right)\right)^{\frac{1}{n^{\star \star}}}}}{\sum_{i} p_{i} \frac{\partial\left(\hat{Z}_{i}\right)^{-1}}{\partial r}(r)} .
$$

REMARK 4.4. The case of power laws for a multiphase thermoviscous heterogeneous material made of periodic layers.

Let us complete the previous Remark by an explicit example.

In the setting of Remark 4.3. consider the case where the thermoviscous material is made of periodic layers of $I$ phases with volume fractions $p_{i}$, which are characterized by heat coefficients $c_{i}(s)$ and by viscosity coefficients $\mu_{i}(s)$ which satisfy hypotheses (2.11), 
(2.12) and (2.15), (2.16) respectively, and which are given by power laws in some interval $A \leq s \leq B$ of $\mathbf{R}^{+}$, i.e. which satisfy

$$
\begin{aligned}
& c_{i}(s)=K_{i} s^{\xi_{i}}, \quad \forall s \quad \text { with } \quad A \leq s \leq B, \\
& \mu_{i}(s)=M_{i} s^{\lambda_{i}}, \quad \forall s \quad \text { with } \quad A \leq s \leq B,
\end{aligned}
$$

where $K_{i}$ and $M_{i}$ are given in $\mathbf{R}^{+}$, where $\xi_{i}$ and $\lambda_{i}$ are given in $\mathbf{R}$ and where $A$ and $B$ are given with $0<A<B<+\infty$. We will finally assume that the powers $\xi_{i}, \lambda_{i}$ and the initial temperature $\theta^{\star \star}$ satisfy

$$
\begin{gathered}
\left(1+\xi_{i}\right) n^{\star \star}+\lambda_{i} \neq 0 \quad \forall i, \\
\theta^{\star \star} \leq A .
\end{gathered}
$$

These two hypotheses are not essential.

In this example the function $\hat{Z}_{i}(s)$ defined by (4.11) is given by

$$
\hat{Z}_{i}(s)=\hat{g}_{i} s^{\frac{\left(1+\xi_{i}\right) n^{\star \star}+\lambda_{i}}{n^{\star \star}}}-\hat{K}_{i}, \quad \forall s \quad \text { with } \quad A \leq s \leq B
$$

(in the case where hypothesis (4.17) does not hold true, the power has to be replaced by a logarithm), where the constants $\hat{g}_{i}$ and $\hat{K}_{i}$ are given by

$$
\begin{gathered}
\hat{g}_{i}=\frac{n^{\star \star}}{\left(1+\xi_{i}\right) n^{\star \star}+\lambda_{i}} K_{i} M_{i}^{\frac{1}{n^{\star \star}}}, \\
\hat{K}_{i}=\hat{g}_{i} A^{\frac{\left(1+\xi_{i}\right) n^{\star \star \star}+\lambda_{i}}{n^{\star \star}}}-\int_{\theta^{\star \star}}^{A} c_{i}\left(s^{\prime}\right)\left(\mu_{i}\left(s^{\prime}\right)\right)^{\frac{1}{n^{\star \star}}} d s^{\prime} .
\end{gathered}
$$

We will assume, moreover, that there exist $C$ and $D$ such that

$$
\max _{i} \hat{Z}_{i}(A) \leq C<D \leq \min _{i} \hat{Z}_{i}(B) .
$$

Then, following parallel lines with the viscoplastic case, we find that the homogenized viscosity coefficient $\mu^{0}$ reads as

$$
\begin{gathered}
\frac{1}{\left(\mu^{0}\left(\hat{Y}^{0}(r)\right)\right)^{\frac{1}{n^{\star \star}}}}=\sum_{i} \frac{p_{i}}{M_{i}^{\frac{1}{n^{\star \star}}}\left(\frac{1}{\hat{g}_{i}}\left(r+\hat{K}_{i}\right)\right)^{\frac{\lambda_{i}}{\left(1+\xi_{i}\right) n^{\star \star}+\lambda_{i}}}}, \\
\forall r \quad \text { with } \quad C \leq r \leq D,
\end{gathered}
$$

and the homogenized heat coefficient $c^{0}$ reads as

$$
\begin{gathered}
c^{0}\left(\hat{Y}^{0}(r)\right)=\frac{\sum_{i} \frac{p_{i}}{M_{i}^{\frac{1}{n^{\star \star}}}\left(\frac{1}{\hat{g}_{i}}\left(r+\hat{K}_{i}\right)\right)^{\frac{\lambda_{i}}{\left(1+\xi_{i}\right) n^{\star \star}+\lambda_{i}}}}}{\sum_{i} p_{i} \frac{n^{\star \star}}{\left(1+\xi_{i}\right) n^{\star \star}+\lambda_{i}} \frac{1}{\hat{g}_{i}}\left(\frac{1}{\hat{g}_{i}}\left(r+\hat{K}_{i}\right)\right)^{-\frac{\xi_{i} n^{\star \star}+\lambda_{i}}{\left(1+\xi_{i}\right) n^{\star \star}+\lambda_{i}}}} \\
\forall r \quad \text { with } \quad C \leq r \leq D .
\end{gathered}
$$

Observe that power laws are not $\mathrm{SbH}$. 
5. Proof of propositions. We can write both (3.8) and (4.6) in the form

$$
d^{\varepsilon}\left(x, u^{\varepsilon}\right) \frac{\partial u^{\varepsilon}}{\partial t}=\frac{|\sigma|^{\xi} \sigma^{\delta}}{\psi^{\varepsilon}\left(x, u^{\varepsilon}\right)},
$$

where

$$
\begin{gathered}
d^{\varepsilon}=\left\{\begin{array}{c}
1 \text { if } u^{\varepsilon}=\gamma^{\varepsilon}, \\
c^{\varepsilon}\left(x, \theta^{\varepsilon}\right) \text { if } u^{\varepsilon}=\theta^{\varepsilon},
\end{array}\right. \\
\xi=\left\{\begin{array}{l}
\frac{1}{n^{*}}-1 \quad \text { if } u^{\varepsilon}=\gamma^{\varepsilon}, \\
\frac{1}{n^{*}}+1 \text { if } u^{\varepsilon}=\theta^{\varepsilon},
\end{array}\right. \\
\delta=\left\{\begin{array}{ccc}
1 & \text { if } u^{\varepsilon}=\gamma^{\varepsilon}, \\
0 & \text { if } u^{\varepsilon}=\theta^{\varepsilon},
\end{array}\right. \\
\psi^{\varepsilon}=\left\{\begin{array}{lll}
\nu^{\varepsilon} & \text { if } u^{\varepsilon}=\gamma^{\varepsilon}, \\
\mu^{\varepsilon} & \text { if } & u^{\varepsilon}=\theta^{\varepsilon} .
\end{array}\right.
\end{gathered}
$$

Let us define the function $\hat{Z}^{\varepsilon}: \Omega \times \mathbf{R} \rightarrow \mathbf{R}$ by

$$
\hat{Z}^{\varepsilon}(x, s)=\int_{u_{0}(x)}^{s} d^{\varepsilon}\left(x, s^{\prime}\right)\left(\psi^{\varepsilon}\left(x, s^{\prime}\right)\right)^{\frac{1}{n^{\star}(x)}} d s^{\prime} \quad \text { in } \Omega \times \mathbf{R} .
$$

We have in particular

$$
\frac{\partial \hat{Z}^{\varepsilon}}{\partial s}(x, s)=d^{\varepsilon}\left(x, s^{\prime}\right)\left(\psi^{\varepsilon}(x, s)\right)^{\frac{1}{n^{\star}(x)}} \quad \text { in } \Omega \times \mathbf{R} .
$$

For a.e. $x \in \Omega$ fixed, the function $s \in \mathbf{R} \rightarrow \hat{Z}^{\varepsilon}(x, s) \in \mathbf{R}$ is one-to-one, strictly increasing and Lipschitz continuous with

$$
0<\alpha^{\zeta+1 / \bar{\beta}} \leq \frac{\partial \hat{Z}^{\varepsilon}}{\partial s}(x, s) \leq \beta^{\zeta+1 / \bar{\alpha}}<+\infty \quad \text { in } \Omega \times \mathbf{R},
$$

where $\alpha, \beta, \bar{\alpha}$ and $\bar{\beta}$ appear in (2.12), (2.16) and (2.18) and $\zeta=0$ if $u^{\varepsilon}=\gamma^{\varepsilon}, \zeta=1$ if $u^{\varepsilon}=\theta^{\varepsilon}$. Therefore for a.e. $x \in \Omega$ fixed, this function has a reciprocal function $r \in \mathbf{R} \rightarrow\left(\hat{Z}^{\varepsilon}\right)^{-1}(x, r) \in \mathbf{R}$ which is also one-to-one, strictly increasing and Lipschitz continuous with

$$
0<\frac{1}{\beta^{\zeta+1 / \bar{\alpha}}} \leq \frac{\partial\left(\hat{Z}^{\varepsilon}\right)^{-1}}{\partial r}(x, r) \leq \frac{1}{\alpha^{\zeta+1 / \bar{\beta}}}<+\infty \quad \text { in } \Omega \times \mathbf{R} .
$$

Then, since

and since

$$
\frac{\partial \hat{Z}^{\varepsilon}}{\partial s}\left(x, u^{\varepsilon}\right) \frac{\partial u^{\varepsilon}}{\partial t}=\left|\sigma^{\star}\right|^{\xi} \sigma^{\delta} \quad \text { in } Q
$$

$$
\hat{Z}^{\varepsilon}\left(x, u_{0}(x)\right)=0 \quad \text { in } \Omega
$$

we have

$$
\hat{Z}^{\varepsilon}\left(x, u^{\varepsilon}(t, x)\right)=\hat{K}^{\star}(t, x) \quad \text { in } Q,
$$


where $\hat{K}^{\star}=\hat{K}^{\star}(t, x)$ is defined by

$$
\begin{gathered}
\frac{\partial \hat{K}^{\star}}{\partial t}=\left|\sigma^{\star}\right|^{\xi} \sigma^{\delta} \quad \text { in } Q, \\
\hat{K}^{\star}(0, x)=0 \quad \text { in } \Omega .
\end{gathered}
$$

Equation (5.9) is equivalent to

$$
u^{\varepsilon}(t, x)=\left(\hat{Z}^{\varepsilon}\right)^{-1}\left(x, \hat{K}^{\star}(t, x)\right) \quad \text { in } Q .
$$

We finally define the function $\hat{\pi}^{\varepsilon}: \Omega \times \mathbf{R} \rightarrow \mathbf{R}$ by

$$
\hat{\pi}^{\varepsilon}(x, r)=\frac{1}{\left(\psi^{\varepsilon}\left(x,\left(\hat{Z}^{\varepsilon}\right)^{-1}(x, r)\right)\right)^{\frac{1}{n^{\star}(x)}}} \text { in } \Omega \times \mathbf{R} .
$$

Observe that in view of (2.16) and (2.18), one has

$$
\frac{1}{\beta^{1 / \bar{\alpha}}} \leq \hat{\pi}^{\varepsilon}(x, r) \leq \frac{1}{\alpha^{1 / \bar{\beta}}} \quad \text { in } \Omega \times \mathbf{R} .
$$

In view of (2.15), (2.16), (2.18) and (5.8), we deduce from (5.13) that

$$
\begin{aligned}
& \left|\frac{\partial \hat{\pi}^{\varepsilon}}{\partial r}(x, r)\right| \\
& =\frac{1}{n^{\star}(x)} \frac{1}{\left(\psi^{\varepsilon}\left(x,\left(\hat{Z}^{\varepsilon}\right)^{-1}(x, r)\right)\right)^{\frac{1}{n^{\star}(x)}}+1}\left|\frac{\partial \psi^{\varepsilon}}{\partial u}\left(x,\left(\hat{Z}^{\varepsilon}\right)^{-1}(x, r)\right)\right|\left|\frac{\partial\left(\hat{Z}^{\varepsilon}\right)^{-1}}{\partial r}(x, r)\right| \\
& \leq \frac{1}{\bar{\alpha}} \frac{1}{\alpha^{1+1 / \bar{\beta}}} M \frac{1}{\alpha^{\delta+1 / \bar{\beta}}} \quad \text { in } \Omega \times \mathbf{R} .
\end{aligned}
$$

Since the functions $r \in \mathbf{R} \rightarrow\left(\hat{Z}^{\varepsilon}\right)^{-1}(x, r) \in \mathbf{R}$ and $r \in \mathbf{R} \rightarrow \hat{\pi}^{\varepsilon}(x, r) \in \mathbf{R}$ are (uniformly in $x$ and $\varepsilon$ ) Lipschitz continuous (see (5.8) and (5.15)), and since the functions $x \in \Omega \rightarrow\left(\hat{Z}^{\varepsilon}\right)^{-1}(x, r) \in \mathbf{R}$ and $x \in \Omega \rightarrow \hat{\pi}^{\varepsilon}(x, r) \in \mathbf{R}$ are measurable and bounded (uniformly in $\varepsilon$ ) for every $r \in \mathbf{R}$ fixed, a well-known lemma in homogenization theory (see, e.g., Lemma 3.8 in Charalambakis and Murat (2006b)) asserts that one can extract a subsequence $\varepsilon^{\prime}$ and that there exist two functions $\hat{Y}^{0}(x, r): \Omega \times \mathbf{R} \rightarrow \mathbf{R}$ and $\hat{\pi}^{0}(x, r)$ : $\Omega \times \mathbf{R} \rightarrow \mathbf{R}$ (which are also Lipschitz continuous in $r$ uniformly in $x$, measurable in $x$ and bounded for every $r \in \mathbf{R}$ fixed), such that for every $r \in \mathbf{R}$ fixed

$$
\begin{gathered}
\left(\hat{Z}^{\varepsilon^{\prime}}\right)^{-1}(x, r) \rightarrow \hat{Y}^{0}(x, r) \quad \text { in } L^{\infty}(\Omega) \text { weak-star, } \\
\hat{\pi}^{\varepsilon^{\prime}}(x, r) \rightarrow \hat{\pi}^{0}(x, r) \quad \text { in } L^{\infty}(\Omega) \text { weak-star. }
\end{gathered}
$$

Moreover, the same lemma asserts that for this subsequence $\varepsilon^{\prime}$, one has

$$
\begin{gathered}
\left(\hat{Z}^{\varepsilon^{\prime}}\right)^{-1}\left(x, \hat{K}^{\star}(t, x)\right) \rightarrow \hat{Y}^{0}\left(x, \hat{K}^{\star}(t, x)\right) \quad \text { in } L^{\infty}(Q) \text { weak-star, } \\
\hat{\pi}^{\varepsilon^{\prime}}\left(x, \hat{K}^{\star}(t, x)\right) \rightarrow \hat{\pi}^{0}\left(x, \hat{K}^{\star}(t, x)\right) \text { in } L^{\infty}(Q) \text { weak-star. }
\end{gathered}
$$

Since $\hat{Z}^{\varepsilon}\left(x, u^{0}(x)\right)=0$, we have

$$
\left(\hat{Z}^{\varepsilon}\right)^{-1}(x, 0)=u_{0}(x) \quad \text { in } \Omega,
$$

and therefore

$$
\hat{Y}^{0}(x, 0)=u_{0}(x) \quad \text { in } \Omega .
$$


Since for every $r, r^{\prime} \in \mathbf{R}$ with $r \geq r^{\prime}$ we have (see (5.8))

$$
\frac{1}{\beta^{\delta+1 / \bar{\alpha}}}\left(r-r^{\prime}\right) \leq\left(\hat{Z}^{\varepsilon}\right)^{-1}(x, r)-\left(\hat{Z}^{\varepsilon}\right)^{-1}\left(x, r^{\prime}\right) \leq \frac{1}{\alpha^{\delta+1 / \bar{\beta}}}\left(r-r^{\prime}\right) \quad \text { in } \Omega,
$$

we also have, for every $r, r^{\prime} \in \mathbf{R}$ with $r \geq r^{\prime}$,

$$
\frac{1}{\beta^{\delta+1 / \bar{\alpha}}}\left(r-r^{\prime}\right) \leq \hat{Y}^{0}(x, r)-\hat{Y}^{0}\left(x, r^{\prime}\right) \leq \frac{1}{\alpha^{\delta+1 / \bar{\beta}}}\left(r-r^{\prime}\right) \quad \text { in } \Omega,
$$

which proves that for a.e. $x \in \Omega$ fixed, the function $r \in \mathbf{R} \rightarrow \hat{Y}^{0}(x, r) \in \mathbf{R}$ is one-to-one, strongly increasing and Lipschitz continuous. Therefore this function has a reciprocal function $s \in \mathbf{R} \rightarrow\left(\hat{Y}^{0}\right)^{-1}(x, s) \in \mathbf{R}$ with the same properties. We define the function $\hat{Z}^{0}: \Omega \times \mathbf{R} \rightarrow \mathbf{R}$ by

$$
\hat{Z}^{0}(x, s)=\left(\hat{Y}^{0}\right)^{-1}(x, s) \text { in } \Omega \times \mathbf{R} .
$$

We also define the function $\psi^{0}: \Omega \times \mathbf{R} \rightarrow \mathbf{R}$ by

$$
\psi^{0}(x, s)=\frac{1}{\left(\hat{\pi}^{0}\left(x, \hat{Z}^{0}(x, s)\right)\right)^{n^{\star}(x)}} \quad \text { in } \Omega \times \mathbf{R},
$$

which is equivalent to (compare with (5.13) $)$

$$
\hat{\pi}^{0}(x, r)=\frac{1}{\left(\psi^{0}\left(x,\left(\hat{Z}^{0}\right)^{-1}(x, r)\right)\right)^{\frac{1}{n^{\star}(x)}}} \quad \text { in } \Omega \times \mathbf{R} .
$$

Since the function $\hat{\pi}^{0}(x, r)$ is bounded from below and from above by strictly positive constants (this is easily deduced from (5.14) and (5.17)), the function $\psi^{0}(x, s)$ satisfies (2.16) (with constants $\alpha$ and $\beta$ possibly different). On the other hand, since the function $\hat{Z}^{0}(x, s)$ is Lipschitz continuous in $s$ uniformly in $x$ (see (5.20) and (5.21)) and since the function $\hat{\pi}^{0}(x, r)$ is Lipschitz continuous in $r$ uniformly in $x$ (this can easily be deduced from (5.15) and (5.17)), the function $\psi^{0}(x, s)$ defined by (5.22) satisfies (2.15) (with a constant $M$ possibly different).

Since (5.12) asserts that $u^{\varepsilon}(t, x)=\left(\hat{Z}^{\varepsilon}\right)^{-1}\left(x, \hat{K}^{\star}(t, x)\right)$, we deduce from (5.18) and (5.21) that

$$
u^{\varepsilon^{\prime}} \rightarrow u^{0} \quad \text { in } L^{\infty}(Q) \text { weak-star }
$$

where

$$
u^{0}(t, x)=\left(\hat{Z}^{0}\right)^{-1}\left(x, \hat{K}^{\star}(t, x)\right) .
$$

On the other hand, we deduce from (5.12), (5.13), (5.19), (5.23) and (5.25) that

$$
\frac{1}{\left(\psi^{\varepsilon^{\prime}}\left(x, u^{\varepsilon^{\prime}}(t, x)\right)\right)^{\frac{1}{n^{\star}(x)}}} \rightarrow \frac{1}{\left(\psi^{0}\left(x, u^{0}(t, x)\right)\right)^{\frac{1}{n^{\star}(x)}}} \text { in } L^{\infty}(Q) \text { weak-star. }
$$

Turning back to the constitutive equation, convergence (5.26) implies that

$$
\frac{\partial v^{\varepsilon^{\prime}}}{\partial x} \rightarrow \frac{\partial v^{0}}{\partial x} \text { in } L^{\infty}(Q) \text { weak-star }
$$

where

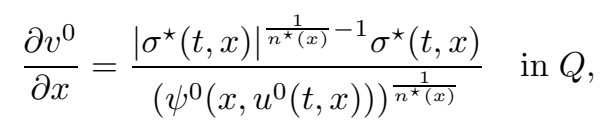


which, thanks to (2.28), is equivalent to

$$
\sigma^{0}=\psi^{0}\left(x, u^{0}\right)\left|\frac{\partial v^{0}}{\partial x}\right|^{n^{\star}(x)-1} \frac{\partial v^{0}}{\partial x} \quad \text { in } Q .
$$

We passed to the limit in (2.4) for both cases. Let us now pass to the limit in (2.3) for the case $u^{\varepsilon}=\theta^{\varepsilon}$.

From the function $\hat{Z}^{0}$ defined by (5.16) and (5.21), and from the function $\psi^{0}$ defined by (5.22), we define the function $d^{0}: \Omega \times \mathbf{R} \rightarrow \mathbf{R}$ by

$$
d^{0}(x, s)=\frac{\frac{\partial \hat{Z}^{0}}{\partial s}(x, s)}{\left(\psi^{0}(x, s)\right)^{\frac{1}{n^{\star}(x)}}} \quad \text { in } \Omega \times \mathbf{R},
$$

or equivalently by

$$
\frac{\partial \hat{Z}^{0}}{\partial s}(x, s)=d^{0}(x, s)\left(\psi^{0}(x, s)\right)^{\frac{1}{n^{\star}(x)}} \quad \text { in } \Omega \times \mathbf{R}
$$

(compare with (5.7)). Since the functions $\frac{\partial \hat{Z}^{0}}{\partial s}(x, s)$ and $\psi^{0}(x, s)$ are bounded from below and from above by strictly positive constants, the function $d^{0}(x, s)$ is correctly defined by (5.28) and satisfies (2.12) (with constants $\alpha$ and $\beta$ possibly different). Moreover, the function $d^{0}(x, s)$ defined by (5.28) satisfies (2.11) (with a constant $M$ possibly different); indeed we have proved above that the function $\psi^{0}(x, s)$ is Lipschitz continuous in $s$, and it can be proved that the function $\frac{\partial \hat{Z}^{0}}{\partial s}(x, s)$ is Lipschitz continuous in $s$ uniformly in $x$. This is due to the fact that $\frac{\partial \hat{Z}^{\varepsilon}}{\partial s}(x, s)$ is Lipschitz continuous in $s$ uniformly in $x$ and $\varepsilon$ (see (5.7), (2.11), (2.15) and (2.18) ), and can be proved by a proof similar to the proof used to prove the similar property for the function $M^{0}(x, s)$ defined in Charalambakis and Murat (2006b) (see the proof of (3.45) in that paper).

On the other hand, (5.10) and (5.25) imply that

$$
\frac{\partial \hat{Z}^{0}\left(x, u^{0}(t, x)\right)}{\partial t}=\left|\sigma^{\star}\right|^{\xi} \sigma^{\delta} \quad \text { in } Q .
$$

Combining (5.29) and (5.30) yields

$$
d^{0}\left(x, u^{0}\right)\left(\psi^{0}\left(x, u^{0}\right)\right)^{\frac{1}{n^{\star}(x)}} \frac{\partial u^{0}}{\partial t}=\left|\sigma^{\star}\right|^{\xi} \sigma^{\delta} \quad \text { in } Q,
$$

which using (2.28) and (5.27) is equivalent to

$$
d^{0}\left(x, u^{0}\right) \frac{\partial u^{0}}{\partial t}=\sigma^{0} \frac{\partial v^{0}}{\partial x} \quad \text { in } Q .
$$

We passed to the limit in (2.3) for the case $u^{\varepsilon}=\theta^{\varepsilon}$.

Let us complete this proof by giving another formula, equivalent to (5.28), for the definition of the homogenized heat coefficient $d^{0}$. Writing (5.28) at the point $s=\hat{Y}^{0}(x, r)$ and using the chain rule applied to the identity $\hat{Z}^{0}\left(x,\left(\hat{Y}^{0}(x, r)\right)\right)=r$ yields

$$
d^{0}\left(x, \hat{Y}^{0}(x, r)\right)=\frac{1}{\frac{\partial \hat{Y}^{0}}{\partial r}(x, r)} \frac{1}{\left(\psi^{0}\left(x, \hat{Y}^{0}(x, r)\right)\right)^{\frac{1}{n^{\star}(x)}}} \text { in } \Omega \times \mathbf{R} .
$$


In conclusion, we passed to the limit in (2.3) and (2.4), and therefore proved that in the setting of Propositions 3.1 and 4.1. the problem (2.1) -(2.8) is SbH, with $n^{0}(x)=n^{\star}(x)$, $d^{0}(x, u)$ defined by (5.28) and $\psi^{0}(x, u)$ defined by (5.22). Note that the homogenized coefficients $d^{0}(x, u)$ and $\psi^{0}(x, u)$ satisfy (2.10)-(2.16) (with constants $M, \alpha$ and $\beta$ possibly different).

Acknowledgements. The numerical examples in this paper are due to George Chatzigeorgiou, whose collaboration is gratefully acknowledged. The results of the present work have been obtained during visits made by the first author to the Laboratoire Jacques-Louis Lions of the Université Pierre et Marie Curie (Paris VI) during the year 2006. The support and hospitality of this institution are gratefully acknowledged. The interest and support of UMT are also acknowledged. The authors sincerely thank the reviewer and the editor for their comments that substantially improved the work.

\section{References.}

Aboudi, J., Pindera, M., Arnold, S., 1999. Higher-order theory for functionally graded materials. Composites: Part B 30, 777-832.

Alshits, V., Maugin, G., 2005. Dynamics of multilayers: elastic waves in an anisotropic graded or stratified plate. Wave Motion 41, 357-394. MR2123921|(2005i:74043)

Bansal, Y., Pindera, M., 2003. Efficient reformulation of the thermoelastic higher-order theory for functionally graded materials. Journal of Thermal Stresses 26, 1055-1092.

Bansal, Y., Pindera, M., 2005. A second look at the higher-order theory for periodic multiphase materials. Journal of Applied Mechanics 72, 177-195.

Bardzokas, D., Zobnin, A., 2005. Mathematical modelling of physical processes in composite materials of periodical structures. URSS Editorial.

Batra, R., Love, B., 2006a. Consideration of microstructural effects in the analysis of adiabatic shear bands in a tungsten heavy alloy. International Journal of Plasticity 22, $1858-1878$.

Batra, R., Love, B., 2006b. Determination of effective thermomechanical parameters of a mixture of two elastothermoviscoplastic constituents. International Journal of Plasticity 22, 1026-1061.

Bensoussan, A., Lions, J.-L., Papanicolaou, G., 1978. Asymptotic methods for periodic structures. North Holland. MR503330 (82h:35001)

Cavalcante, M., Marques, S., Pindera, M.-J., 2007. Parametric formulation of the finitevolume theory for functionally graded materials, part-i: analysis. Journal of Applied Mechanics 74(5), 935-945.

Cavalcante, M., Marques, S., Pindera, M.-J., 2008. Computational aspects of the parametric finite-volume theory for functionally graded materials, part-i: analysis. Journal of Computational Materials Science 44(2), 422-438.

Charalambakis, N., Murat, F., 1989. Weak solutions to initial-boundary value problems for the shearing of non-homogeneous thermoviscoplastic materials. Proceedings of the Royal Society of Edinburgh 113A, 257-265. MR1037731|(91b:73008) 
Charalambakis, N., Murat, F., 2006a. Approximation by finite elements, existence and uniqueness for a model of stratified thermoviscoplastic materials. Ricerche di Matematica 55, 171-218. MR2279421 (2007j:35224)

Charalambakis, N., Murat, F., 2006b. Homogenization of stratified thermoviscoplastic materials. Quarterly of Applied Mathematics 64, 359-399. MR2243868 (2007c:74051)

Charalambakis, N., Murat, F., 2009. Stability by homogenization of thermoviscoplastic problems, in press in Mathematical Models and Methods in Applied Sciences.

Francfort, G., Leguillon, D., Suquet, P., 1983a. Homogenization for linearly viscoelastic bodies. Comptes-Rendus de l'Académie des Sciences de Paris I 296, 287-290. MR693795 (84c:73041)

Francfort, G., Nguyen, Q., Suquet, P., 1983b. Thermodynamics and the homogenized thermomechanical behavior. Comptes-Rendus de l'Académie des Sciences de Paris II 296, 1007-1010. MR720431 (84i:73012)

Ghosh, S., Lee, K., Raghavan, P., 2001. A multi-level computational model for multiscale damage analysis in composite and porous materials. International Journal of Solids and Structures 38, 2335-2385.

Guinovart-Diaz, R., Rodriguez-Ramos, R., Bravo-Castillero, J., Maugin, G., 2005. A recursive asymptotic homogenization scheme for multi-phase fiber-reinforced composites. Mechanics of Materials 37, 1119-1131.

Hashin, Z., 1983. Analysis of composite materials: A survey. Journal of Applied Mechanics $50,481-505$.

Idiart, M., Moulinec, H., Ponte-Castaneda, P., Suquet, P., 2006. Macroscopic behavior and field fluctuations in viscoplastic composites: Second-order estimates versus full-field simulations. Journal of the Mechanics and Physics of Solids 54, 1029-1063. MR2216544 (2006k:74016)

Lemaitre, J., Chaboche, J.-L., 2001. Mécanique des matériaux solides. Dunod.

Maugin, G., 1992. The thermomechanics of plasticity and fracture. Cambridge University Press. MR1173212 (93i:73001)

Michel, J., Suquet, P., 2004. Computational analysis of nonlinear composites structures using the nonuniform transformation field analysis. Computer Methods in Applied Mechanics and Engineering 193, 5477-5502. MR2103055 (2005f:74024)

Murat, F., 1977. H-convergence. Séminaire d'analyse fonctionnelle et numérique de l'Université d'Alger. Multicopied, 34 pages. English translation: Murat, F. and Tartar, L. (1977), $H$-convergence, in Topics in the mathematical modelling of composite materials, ed. by A. Cherkaev and R. V. Kohn, Progress in Nonlinear Differential Equations and their Applications 31, pp. 21-43. Birkhäuser.

Pindera, M.-J., Khatam, H., Drago, A., Bansal, Y., 2009. Micromechanics of spatially uniform heterogeneous media: A critical review and emerging approaches. Composites: Part B 40, 349-378.

Sanchez-Palencia, E., 1978. Non-homogeneous media and vibration theory. Lecture Notes in Physics 127, Springer Verlag. MR578345 (82j:35010)

Suquet, P., 1982. Plasticité et homogénéisation. Ph.D. thesis, Université Pierre et Marie Curie (Paris VI). 
Suquet, P., 1983. Limit analysis and homogenization. Comptes-Rendus de l'Académie des Sciences de Paris II 296, 1335-1358. MR720280 (84j:73066)

Suquet, P., 2005. On the effect of small fluctuations in the volume fraction of constituents on the effective properties of composites. Comptes-Rendus de l'Académie des Sciences de Paris, Mécanique 333, 219-266.

Tartar, L., 1977. Homogénéisation et compacité par compensation. Cours Peccot, Collège de France. Partially written in Murat, F. (1977).

Wright, T., 2002. The physics and mathematics of shear bands. Cambridge University Press. MR1916003 (2004g:74014) 